\section{Ban predators from the scientific record}

Predatory journals are threatening the credibility of science. By faking or neglecting peer review, they pollute the scholarly record with fringe or junk science and activist research. I suggest that every publishing stakeholder could contribute to reining in these journals.

Universities and colleges should stop using the quantity of published articles as a measure of academic performance. Researchers and respectable journals should not cite articles from predatory journals, and academic library databases should exclude metadata for such publications.

Companies that supply services to publishers, including those that license journal-management software or provide standard identifiers, should decline to work with predatory publishers.

Scholarly databases such as Scopus and Thomson Reuters Web of Science need to raise the bar for acceptance, eliminating journals and publishers that use flawed peer-review practices. The US National Center for Biotechnology Information should do the same for PubMed and PubMed Central.

Finally, advocates of openaccess publication must stop pretending that the author-pays model is free of serious, longterm structural problems (see J. Beall Nature 489, 179; 2012). Just because it works well in a few cases doesn't mean it always works.

Jeffrey Beall Auraria Library, University of Colorado Denver, USA.

jeffrey.beall@ucdenver.edu

\section{Hail local fieldwork, not just global models}

We contend that science's 'publish-or-perish' culture, which selects for rapid publication in high-ranking journals, has contributed to the demise of field-based studies (see K.-D. Dijkstra Nature 533, 172-174; 2016).

Top-tier journals tend to favour large-scale analyses that answer big, general questions (see J.M. Fitzsimmons and J. H. Skevington Nature 466, 179; 2010), presumably because they help to boost journal impact factors. Unlike basic ecological and observational studies, such analyses seldom involve the collection of new, local field data. Instead, they depend mainly on modelling of published information, often over scales that would be logistically and economically challenging for conventional field investigations.

Because publication in leading journals is science's currency to capture funding, funders also tend to select against field-based research studies - including those with undeniable reach and importance, such as longterm biodiversity monitoring (see T. Birkhead Nature 514, 405; 2014).

Given the current biodiversity crisis, journals and funding agencies - as well as the scientific community - must act to reverse this trend.

Catarina Ferreira Trent University, Peterborough, Canada. C. Antonio Ríos-Saldaña BioCórima, Arteaga, Mexico. Miguel Delibes-Mateos Institute for Advanced Social Studies (IESA-CSIC), Córdoba, Spain. catferreira@gmail.com

\section{A code of conduct for data on epidemics}

As a long-term champion of open-access research data on pandemic viruses and a member of the Italian Parliament, I urge Brazil to hasten the reform of its current biosecurity legislation.

This would enable sharing of vital Zika virus samples and information, as recently called for by the World Health Organization (see M.-P. Kieny et al. Nature 533, 469; 2016, and go.nature.com/1o4x3dp).
Data sharing for viruses has been disappointingly patchy since I first ignited the debate by depositing my unpublished sequence data for $\mathrm{H} 5 \mathrm{~N} 1$ avian influenza virus in a public database, rather than in the established password-protected system (see Nature 440, 255-256; 2006). When the 2009 H1N1 swine flu virus emerged, the importance of data sharing was evident in the rapid response to the pandemic. However, the first isolate of the Middle East respiratory syndrome (MERS) coronavirus from Saudi Arabia was controversially submitted for patenting in 2013 (see go.nature. com/luu7ldd). And in last year's Ebola virus epidemic, there were significant gaps in the availability and posting of online sequence data (N. L. Yozwiak et al. Nature 518, 477-479; 2015).

To overcome such hurdles, I suggest that the United Nations and relevant stakeholders should develop guidelines for scientists, institutions and governments.

These should harmonize codes of conduct on sharing information about emerging biological threats - including pathogens that are resistant to antimicrobials.

Ilaria Capua Italian Chamber of Deputies, Italy.

ilariacapua1@gmail.com

\section{Archive computer code with raw data}

As the leader of a young research group, I recognize the need to archive more than just the raw data that underpin scientific papers. Archiving computer code is also important for safeguarding scientific integrity and for facilitating ongoing projects.

Most scientific journals demand that researchers make their primary data publicly available in the interest of reproducibility. Access to the associated computer code enables statistical analyses and calculations to be validated (see Nature 514, 536; 2014).
The more explicit the links between the data, the code and the resulting outputs (including tables and figures), the easier it is to reproduce the findings.

Software tools such as knitr and R Markdown allow the description and code of a statistical analysis to be combined into a single document, providing a pipeline from the raw data to the final results and figures. Outputs are updated by re-running the scripts using version-control tools such as Git and GitHub.

My group has elected to use these tools and to include R Markdown files as supplementary information to our publications (see, for example, M. A. Stoffel et al. Proc. Natl Acad. Sci. USA 112, E5005-E5012; 2015). I suggest that journals encourage this practice to help to fight the reproducibility crisis.

Joseph I. Hoffman University of Bielefeld, Germany.

joseph.hoffman@uni-bielefeld.de

\section{Tea but not dinner with Karl von Frisch}

In the 1960s, I had reason to discuss with my friend the late Annemarie Weber, a muscle physiologist, the morality of ethologist Karl von Frisch's decision to continue his studies on honeybee communication during the Second World War (see M. L. Winston Nature 533, 32-33; 2016).

Annemarie's father, Hans Weber, had been removed from his post as professor at the University of Tübingen because he was an opponent of the Nazis. Too famous to be harmed, he was instead transferred to a minor university in East Prussia. Her precise but nuanced response to me was: "After the war, my father would have tea with von Frisch - but dinner, never."

Michael Katz March of Dimes

Foundation, White Plains, New York, USA.

mkatz@marchofdimes.org 